\title{
ASSESSING THE IMPACT OF ABSTRACT REPRESENTATIONS AND REFRAMING OF DESIGN BRIEF INFORMATION ON CREATIVE IDEATION
}

\author{
Kang, Jacob Kai Siang (1,2); Meurzec, Rianne Wally (1); Chia, Pei Zhi (1); Wood, Kristin L. (1,2); \\ Koronis, Georgios (1,2); Silva, Arlindo (1,2)
}

1: Singapore University of Technology and Design; 2: SUTD-MIT International Design Centre (IDC, idc.sutd.edu.sg)

\begin{abstract}
The overarching goal of this work is to support creative ideation in engineering design with the aim of overcoming design fixation. We study the impact of abstract representations and ways to frame the problem in design briefs on the creativity of concept sketches. Framing/Reframing involves shifting perspectives on the design purpose and to reveal insights and opportunities. Two Framing/Reframing techniques are tested: the Ishikawa/Fishbone Diagram to identify root causes and a blend of Parnes' Restatement/SCAMPER method to encourage divergence in problem perception. Abstract representations of requirements were used as stimuli to foster transfer and associative thinking. Using a full-factorial experimental design with brief variations, C-Sketch ideas developed by first-year engineering/architecture students were evaluated for their creativity. Our results showed a positive interaction effect for novelty and usefulness when the Fishbone Reframing method was used with abstract representation, but there was no difference in creativity scores when comparing the two Framing/Reframing methods between each other.
\end{abstract}

Keywords: Creativity, Conceptual design, Collaborative design, Requirements, New product development

\section{Contact:}

Kang, Jacob Kai Siang

Singapore University of Technology and Design

International Design Center

Singapore

Jacob_kang@mymail.sutd.edu.sg

Cite this article: Kang, J.K.S., Meurzec, R.W., Chia, P.Z., Wood, K.L., Koronis, G., Silva, A. (2019) 'Assessing the

Impact of Abstract Representations and Reframing of Design Brief Information on Creative Ideation', in Proceedings of the 22nd International Conference on Engineering Design (ICED19), Delft, The Netherlands, 5-8 August 2019. DOI:10.1017/dsi.2019.31 


\section{INTRODUCTION}

This study is motivated by Framing/Reframing methods and abstract representations to overcome fixation and improve creative ideation. The way in which a design problem-opportunity is framed and defined charts the design direction and influences ideation outcomes (Getzels, 1975). We use the term "problem-opportunity" as the designer's response to a design brief; to perceive problems as opportunities. Framing/Reframing a problem opportunity involves sensemaking (Valkenburg, 2000), identifying surprising observations and interactions, challenging assumptions, deducing insights and reflecting (Schön, 1983). Additionally, we infer new possibilities by formulating new problemopportunities from other contexts. The act of Framing/Reframing has been shown in studies to benefit design cognition and ideation (Linsey et al., 2008, Moreno et al., 2016). More abstract functional representations have also been shown to be effective as stimuli in reducing fixation (Studer et al., 2016) and fostering the generation of new ideas (Linsey et al., 2010, Zahner et al., 2010). Through the analysis of the novelty and usefulness of the Collaborative Sketching (C-Sketch) (Shah et al., 2011) with variations of briefs based on different factor settings, this study aims to contribute to the field of creativity as an empirical study of the effect of Framing/Reframing methods, specifically with the use of the Ishikawa/Fishbone Diagrams, Parnes' Restatement Method and abstract representations on ideation in the engineering design context.

\section{BACKGROUND}

\subsection{Creativity and ideation definition}

Ideation involves the production of original and feasible ideas to solve a problem (Bourgeois-Bougrine et al., 2017). Ideas in the form of products or various modal responses are deemed creative when they are both novel and appropriate (i.e. useful, correct, valuable) (Amabile, 1996). Novelty is defined as the extent to which the design is different from usual designs. Novel designs may not be valuable or apply to the problem if they are not feasible and useful; therefore, we define usefulness as the degree to which the design's ability to be implemented (i.e. feasibility) and its ability to solve the problem (effectiveness) (Dean, 2006).

\subsection{Design brief}

A design brief is a document that states the aims and desired results of a design project. To address the brief, the designer must first understand the design context, determine the purpose and priorities and "impose a coherence that guides subsequent moves" (Schön, 1988). Situated at the beginning of the design process (Camburn et al., 2017), Framing influences the response to the design brief, ideation and project direction.

\subsection{Use of abstract representations and impact on ideation}

Concrete terms refer to detailed, explicit examples of things that can be directly perceived by our senses, while abstract terms refer to ideas, attributes and relationships requiring inferences using mental representations from language and context (Marschark and Paivio, 1977). The impact of abstract representations on creativity is a topic of interest across disciplines. In pedagogy, the transfer of ideas between domains using graphics simulations can be improved by presenting problems in an abstract, idealised form (Goldstone and Sakamoto, 2003). In the design of information systems, abstraction and re-representation has been reported to improve novelty while reducing appropriateness of ideas (Zahner et al., 2010). In engineering design literature, findings suggest that abstract brief requirements and analogies improve idea associations between domains (Linsey, 2007, Linsey et al., 2012).

On the one hand, when problems are too concrete and specific, transfer becomes difficult; on the other hand, without any "domain instantiation," problems represented too abstractly reduce successful outcomes (Holyoak and Thagard, 1989). A good abstract representation, according to Zahner, "will conform to problem constraints while increasing the range of associations and domains" (Zahner et al., 2010). Of the studies reviewed, the use of abstract stimuli improved novelty by reducing detailed specifications and replacing them with more general function-based terminologies in the domain. For 
instance, to reduce fixation in the design problem description, more abstract functions expressed as troponyms (changing "action verbs") and hypernyms (generic action terms) such as "transmitting energy to object or separating outer structure from inner material" were used instead of tangible, concrete terms expressed as hyponyms (specific terms) like "using a press to crush and remove the peanut shell" (Heckler, 2010, Linsey et al., 2010). Hence, in addition to the control brief, for groups with abstract representations as stimuli, we express a brief's requirements as abstract representations in the language of simple, generic scientific/physics principles that engineering students can relate to, with the intention to improve association and transfer as shown in Table 1.

Table 1. Comparisons between concrete and abstract representation of design needs/requirements

\begin{tabular}{|c|c|}
\hline Control Brief & Abstract Representation as Stimuli \\
\hline Design a device to & Design a way of \\
\hline $\begin{array}{l}\text { Help the user move independently across } \\
\text { difficult, uneven, narrow, inclined terrains. }\end{array}$ & Transferring energy from system or device to people with \\
little or no energy \\
\cline { 2 - 2 } $\begin{array}{c}\text { Support movement of user onto and off the } \\
\text { device (support daily living). }\end{array}$ & Transferring signals from person to system or device \\
\cline { 2 - 2 } & Effecting a controlled displacement of an object in any \\
& axis \\
\hline Be affordable & Acquiring within a person's resource capability \\
\hline Be easy to maintain and repair & Restoring something to its original state \\
\hline Be easy to store or move when not in & Transforming an entity to fit storage dimensions \\
\hline
\end{tabular}

\subsection{Framing and reframing and impact on ideation}

Studying a designer's response to design briefs, our research aims to collect evidence to test if the active reflection, shifting of perspectives and resolution of a problem frame through the Reframing process improves the novelty and usefulness of designs. After all, "the way a problem is posed is the way the dilemma will be resolved" (Getzels, 1975). Multiple, alternative representations in meaningful forms are the goal. Questions and insights open new ideation avenues and encourage the use of Framing/Reframing to see problems as opportunities. Based on protocol studies of industrial designers, a problem driven design strategy, i.e. more emphasis on defining the problem, rather than a solution-driven strategy (i.e. a focus on generating solutions), produced better results in solution quality and novelty (Kruger and Cross, 2006). While there are theoretical claims to Framing/Reframing's effectiveness, few empirical studies are carried out on the effect of Framing/Reframing in Design (Getzels and Csikszentmihalyi, 1977, Studer et al., 2016). This current study intends to be a first step at understanding the cognitive processes and responses of students to design briefs by finding the preferred combination for Framing/Reframing methods and abstract stimuli and the impact on creativity of ideas. For Framing, specifically for problem discovery, methods include Kepner Tregnoe Problem Analysis; "identifying and evaluating potential causes by understanding what, where, when and extent is or is not" (Kepner and Tregoe, 2013), Dunckler Diagrams; obtaining the "real" problem by moving from present states to desired states (Fogler and LeBlanc, 2008) and asking "Five Whys'(Kohfeldt and Day Langhout, 2012). In common, these methods involve understanding context and finding true problems. A pilot study with corporate clients using "Five Whys" helped the research team realise that if the initial "Why" was framed poorly at first, the additional "Whys" asked strayed from context and became irrelevant. Also, there may be confusion caused by the categories of "is/is not" in Kepner Tregoe Problem Analysis and "present/desired states" in Dunckler Diagrams. Thus, Fishbone was chosen as the preferred method of problem discovery to study. Compared to an unguided asking of "whys", clear categories in the Fishbone diagram to think about the problem's root help guide thought processes strategically. Specifically for reframing using linguistic re-representations, methods surveyed include the WordTree method using design by analogy (Linsey et al., 2012) and Parnes' Restatement (Parnes, 1967). Parnes' Restatement was chosen as it was a simpler process with less time required and was less investigated empirically. Comparing the two Framing/Reframing activities, the fishbone diagram encourages a more analytical, cause-and-effect questioning approach to framing problems and determining root causes before ideation (Figure 1), while Parnes' restatement/SCAMPER encourages radical divergence in the Framing/Reframing through term substitution using triggers of elaboration, substitution and stating opposites of words (Figure 2). 


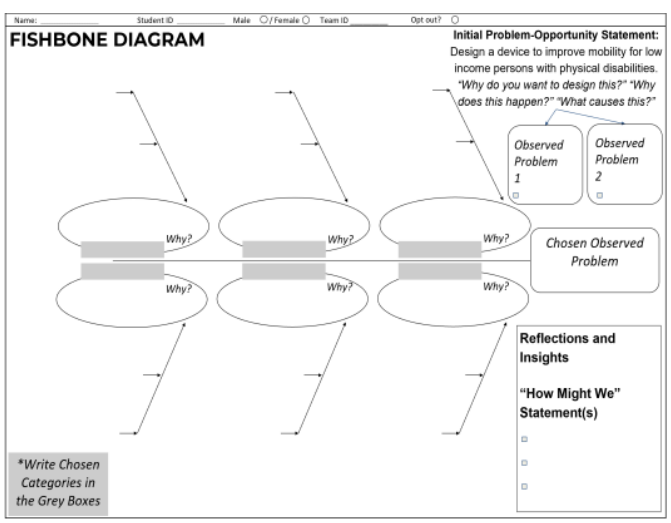

Figure 1. Ishikawa/Fishbone Diagram

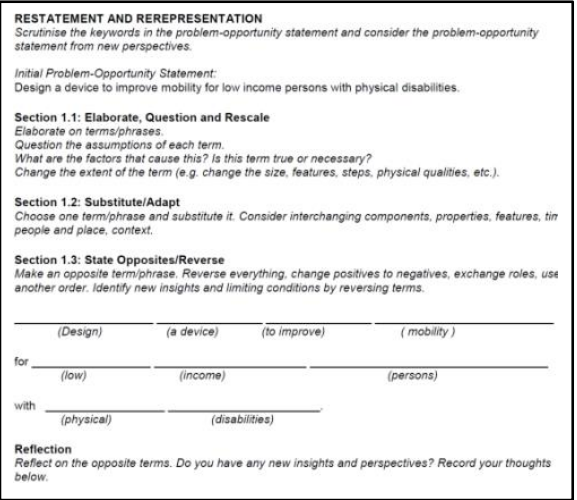

Figure 2. Excerpt of Parnes' Restatement/ SCAMPER handout

\subsection{Framing/reframing - Ishikawa/fishbone diagrams}

First developed by Ishikawa for quality control in manufacturing (Ishikawa, 1982), Fishbone diagrams (Figure 1) are used to identify the underlying/root causes of problems based on observations and cause-and-effect questioning using "Whys". This is helpful when there are barriers to problem-solving and little quantitative data is available. Students designers are first instructed to write observed problems from the initial design brief. After that, they choose from a list of categories to guide the asking of "whys". The categories are multifaceted, including people (stakeholders/users), processes, technology, information, machine, methods, materials, operations, interactions, environment, etc. The use of multiple categories also encourages a more holistic and system-wide approach in the development of product service systems in engineering design and provide the semantic context in which the inquiry is made. Then, the student designers ask "why" several times to find the most likely explanations for the observations and reflect on root causes. This process of probing is an act of abductive reasoning (making the best guess from incomplete information) and expression of modality (operators to qualify a statement) (Josephson, 1994); gathering insights from observed outliers. The fishbone diagram is thus used as a problem framing technique to help identify and focus on critical issues and find relationships between different parts of the problem thus generating insights.

\subsection{Framing/reframing - Parnes' restatement/substitute method in SCAMPER}

Reframing involves shifting lenses on the design problem and purpose given its context (Carlgren et al., 2016). Parnes' restatement (Hicks, 2004) and SCAMPER (Serrat, 2017) are divergence techniques used to trigger varied viewpoints of a statement or frame. In contrast with the Ishikawa/fishbone diagram which is guided more by causal logic to discover a problem's root, this method helps framing/reframing by changing keywords in the problem statement using elaboration, substitution and opposite stating to consider new perspectives. Especially for "substitution" and "stating opposites (antonyms)", terms that are replaced have lower semantic similarity and make less logical sense when read as a sentence. Substituted terms using these triggers are meant to be springboards for "finding true and meaningful constraints and relationships to frame the problem to a more accurate representation" (Parnes, 1967). The first, "Elaborate, Question and Rescale," borrowed from Parnes' requires an elaboration or changing the extent/magnitude of the term. The next trigger, borrowed from SCAMPER, was "Substitute and Adapt." Typically used for ideation, the tool was adapted to reframe the problem by substituting words, components, and properties. The last trigger requested participants to "State Opposites or Reverse Terms," as in used in Parnes' restatement triggers. Following each trigger, student designers were asked to reflect on the new terms and formulate their problem opportunity statement as a "How Might We" question. Parnes' restatement exercise promotes the rapid divergence in problem perspective with associations in multiple directions and may help participants improve the novelty of frames and ideas.

\subsection{The use of "How might we" as a problem opportunity frame}

"How might we" statements are a means of framing and launching ideation. They can help elicit desired projections of outcomes that help generate functional solutions (Higgins et al., 1989). For 
groups exposed to reframing, after completing either the fishbone diagram exercise or the Parnes' restatement exercise, participants were then asked to construct and choose a final problem opportunity "How might we" statement to work on their C-sketch ideation activity. Groups not in reframing categories worked only on the design brief given.

\section{RESEARCH QUESTIONS}

Practically for C-Sketch ideation in an engineering design setting, will individual factors of Framing/Reframing or Abstract Representations alone or an interaction effect of these factors improve novelty and usefulness of concept sketches? Which experimental condition has the best performance? Our research questions are:

RQ1. Comparing Framing/Reframing methods (Fishbone and Parnes' Restatement) with each other and to a control brief, which is more effective in improving novelty and usefulness scores of participants' concept sketches?

RQ2. Using a full factorial design of experiments, what are the main or interaction effects of Abstract Representations and Framing/Reframing methods (Fishbone and Parnes' Restatement) in design briefs on the novelty and usefulness scores of participants' concept sketches?

\section{EXPERIMENTAL METHODS}

The questions above are investigated via a problem-based engineering product design case study conducted over a 2-hour long session. This included a 40-minute reframing exercise for selected classes, C-sketch ideation and Pugh Chart evaluations. The study was part of the University's 2018 Introduction to Design course. A total of 114 first-year university student designers who would choose to specialise in either architecture or engineering in the second year were recruited, resulting in a total of 341 concept sketches. The students were 18-21 years of age and were allocated to classes with an even mix of genders, nationalities, educational backgrounds, academic scores, subject preferences for majors, as well as Myer Briggs Type Indicator and Big Five personality profiles.

We use C-Sketch, an ideation tool known for its effectiveness in promoting ideation (Shah et al., 2011). "Constructive perception" by C-Sketch encourages designers to actively reconfigure sketches and find new meanings and ideas from each other's sketch. (Schön, 1983, Tversky and Suwa, 2009). Participants were grouped into teams of 4-5 students from a total of 6 classes and were tasked with completing a C-Sketch activity, yielding 3 sketches per participant on average. The C-Sketch exercise requires each participant to first spend 10 minutes to create three separate concepts, before rotating their C-Sketch sheet within their group in 5-minute intervals so that other members of the group can amend or improve the original sketches. The process was repeated until each student designer received his/her original sheet. Examples of concept sketches are shown in Figure 3. The design problem was to "design a device to improve mobility for low income persons with physical disabilities." Each class was given the same control brief but a different combination of additional factors in their design brief. The use of both reframing methods as factors in a single full factorial experiment was not possible, and therefore they had to be done in two separate full factorial experiments. This was in consideration of the cognitive load on students that had to do two reframing methods integrated with C-Sketch for approximately 2 hours. For comparing between the two different reframing methods, independent sample t-tests and Kruskal Wallis nonparametric test were used.

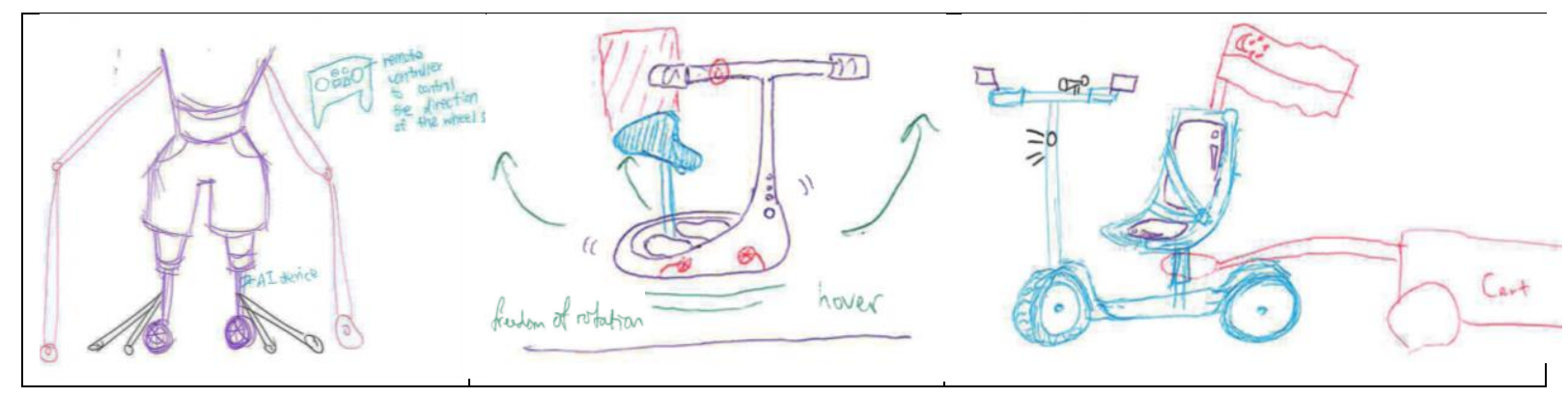

Figure 3. Sketch with high novelty and usefulness scores (left) Sketch with high novelty and low usefulness scores (middle) and Sketch with low novelty and high usefulness scores (right) 


\subsection{Design of experiment - full factorial design}

Most creativity studies adopt a one-variable-at-a-time approach which does not account for possible interactions between variables/factors. A full factorial DOE (Antony, 2014) is an experiment design of factors with discrete levels and sets of samples that have a mix of these levels across factors. This study investigated the effects of two factors: (A) Abstract Representation (abstraction in product's functional requirements), and (R) Reframing on novelty and usefulness shown in Table 2 below.

Table 2. List of factors and factor levels for the two full factorial experiments

\begin{tabular}{cccc}
\hline Factors & Labels & Low Level (-) & High Level (+) \\
\hline $\begin{array}{c}\text { Abstract Representation } \\
\text { Reframing (Parnes' }\end{array}$ & $\mathrm{A}$ & Not present (0) & $\begin{array}{c}\text { Present (1) } \\
\text { Present (1) }\end{array}$ \\
\hline Restatement/SCAMPER) & $\mathrm{R}(\mathrm{pr})$ & Not present (0) & \\
\hline Factors & & & High Level (+) \\
\hline Abstract Representation & Labels & Low Level (-) & Present (1) \\
Reframing (Fishbone) & $\mathrm{A}$ & Not present (0) & Present (1) \\
\hline
\end{tabular}

Two $2^{2}$ full factorial experimental designs were used to test for main and interaction effects on the response variables (i.e. novelty and usefulness scores). Based on the above experimental design, each class received a different combination of factors: 1 class received the control brief, 3 classes received the control brief and one factor $(\mathrm{A}, \mathrm{R}(\mathrm{r})$ or $\mathrm{R}(\mathrm{f}))$ present, and 2 classes received the control brief with two factors $(\mathrm{AR}(\mathrm{r})$ or $\mathrm{AR}(\mathrm{f})$ present).

\subsection{Evaluation of creativity scores}

Sketches were given 1-to-5 scores on Likert-type items, as informed by Amabile's Consensual Assessment Technique (Amabile, 1996). Some of the more widely-used methods to evaluate ideation effectiveness for engineering include novelty, variety, quantity (fluency), quality (Shah et al., 2003), workability, relevance and specificity of ideas (Dean, 2006). While there are many metrics and approaches for evaluating creativity, considering the scale of the study, two metrics, novelty (defined as the extent to which the design is different from usual forms of mobility) and usefulness (Finke, 1990) were chosen because of their simplicity and understandability. Metrics for usability in our previous study resulted in low inter-rater reliability, which seemed to be partially due to the generic phrasing of the usability metric that resulted in differing interpretations between judges. To improve the inter-rater reliability of the judges' scores and encapsulate multiple facets of usefulness (Dean, 2006), we decomposed usefulness into the more clearly defined sub-metrics of implementability (feasibility) and effectiveness, then averaged the scores of the two usefulness sub-metrics so that each sketch had one usefulness score. Factor analysis conducted on the sub-metrics of usefulness yielded a uni-dimensional construct for usefulness. We used multiple student-raters and tested them for interrater agreement. Students were briefed and instructed on the evaluation criteria they were to use for assessment (Table 3), and then the teams swapped sketches so that each team assesses another teams' sketches. While the reliability of relatively inexperienced designers for the evaluation may be a concern (Kaufman et al., 2008), studies showed that it is possible to use such designers to evaluate design creativity with minimal training while still achieving expert-level feedback. (Storme et al., 2014). To check, we took samples of the combined evaluations of 4 student designers (First-year students) and 4 experts (designers with at least 4 years of experience in engineering design) for seven classes to test for inter-rater reliability. The average measures (ICC-2) scores for absolute agreement were in the range of good reliability at 0.60 for novelty scores and 0.60 for usefulness scores.

To evaluate the creativity of the ideas in the sketches, each sketch was scored by four judges, on metrics of novelty and usefulness. Each sketch was rated by different sets of raters. The mean of the four judges was taken for each metric. As there were three sketches per individual, the mean of the three sketches' scores for each metric was then averaged so that individual had one score for each metric. To check inter-rater reliability of four judges, we calculate the intra-class correlation coefficient (ICC-1) estimates for each of the scores based on one-way random effects. ICC estimates were in the range of good reliability at 0.63 for novelty scores and 0.63 for usefulness scores. 
Table 3. Novelty and usefulness evaluation metrics

\begin{tabular}{|l|l|}
\hline Novelty \\
The extent to which the design is different from the usual form of mobility.
\end{tabular}

\section{RESULTS}

\subsection{Independent samples t-test and kruskal wallis (for comparing reframing methods)}

To address RQ1, Minitab statistical software was used to run the independent samples t-test to compare the fishbone diagram and Parnes' restatement groups to determine which sample is more effective at promoting novelty and usefulness. The p-value is $0.782(\alpha=0.05)$ for novelty and $0.493(\alpha=0.05)$ for usefulness; therefore, we accept the null hypothesis that there is no difference in novelty and usefulness scores between the two reframing methods. Comparing two different reframing methods when the factors interact with concreteness fading/abstract stimuli, the $p$-value is $0.739(\alpha=0.05)$ for novelty and 0.487 $(\alpha=0.05)$ for usefulness; therefore, we accept the null hypothesis that there is no difference in novelty and usefulness scores between the two reframing methods even when they are interacting with concretenessfading/abstract stimuli. For novelty scores, as the distribution of the class with the control brief did not meet requirements for a normal distribution, a non-parametric approach was adopted using the independent samples Kruskal Wallis test. After Bonferroni correction with adjusted p-values, none of the pairwise tests are significant, indicating that distributions of creativity scores from class who received reframing briefs compared to control brief are not significantly different. In summary, there appeared to be no difference in the creativity scores between classes that did different reframing techniques and between classes that received reframing briefs to the class that received the control brief.

\subsection{Full factorial analysis}

Assumptions of homoscedascity (Barlett's test) and normality (Shapiro Wilk test) were met for fulfilling requirements for ANOVA. To determine the presence of main and interaction effects, the normal plots of the standardised effects and regression determine the direction, magnitude and importance of the effects. (seen in figure 3). Effects with a red triangular symbol are statistically significant at $\alpha=0.05$ while those with a circle symbol are not statistically significant. For the novelty metric, results show that only the two-way interaction effects of both instances of abstraction with reframing, AR(r) and AR(f) are statistically significant with positive standardised effects (Figure 4). The effect sizes (partial eta squared, $\eta^{2}$ ) of factors AR(f) and AR(r) have a medium to large effect size of 0.062 and 0.100 respectively (Watson, 2009).

Box-Cox Transformation was applied to meet assumptions for normality and homogeneity of variance for usefulness scores for $\operatorname{AR}(f)$. AR(f) is statistically significant with positive standardised effect and small to medium effect size (partial eta squared, $\eta^{2}$ ) of 0.049 (Figure 5). For the Design of Experiment performed on the factors of Abstract Representation and Parnes' Restatement on usefulness (Right of Figure 5), attempts to transform the data could not meet both normality assumptions and homogeneity of variance, thus the results are inconclusive and will not be discussed to avoid false positives. 

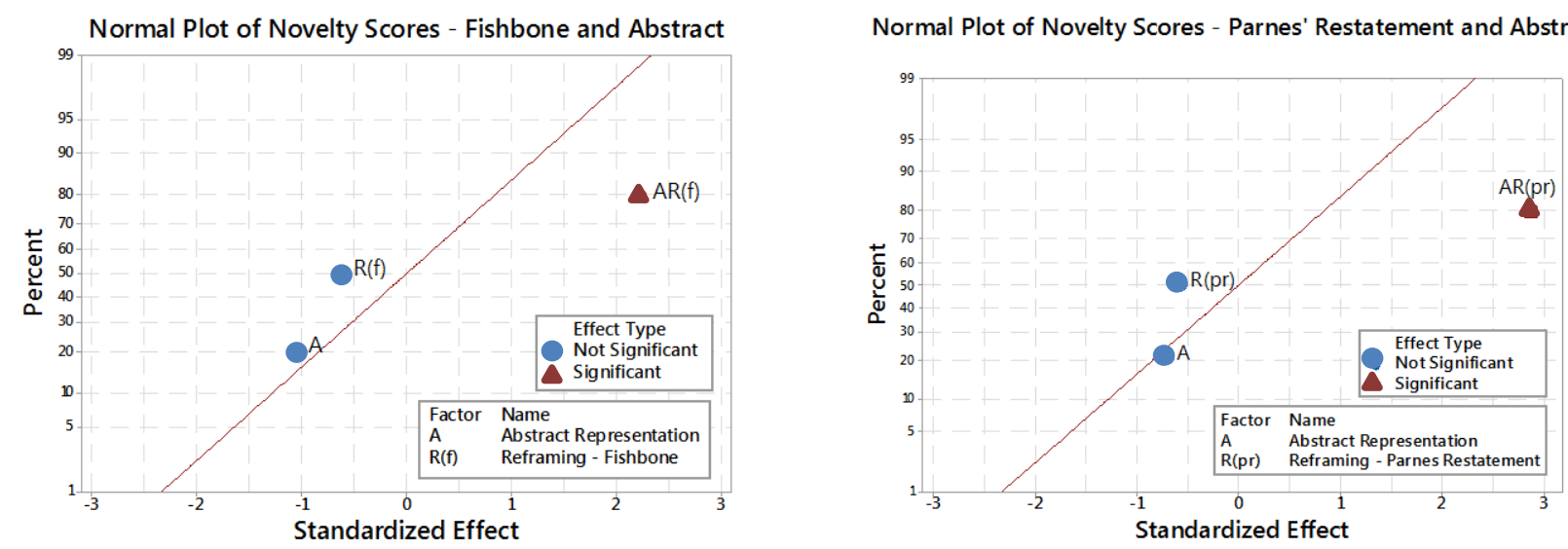

Figure 4. Normal plot of novelty scores for factors Abstract Representation with Fishbone (left) and Abstract Representation with Parnes' Restatement (right).
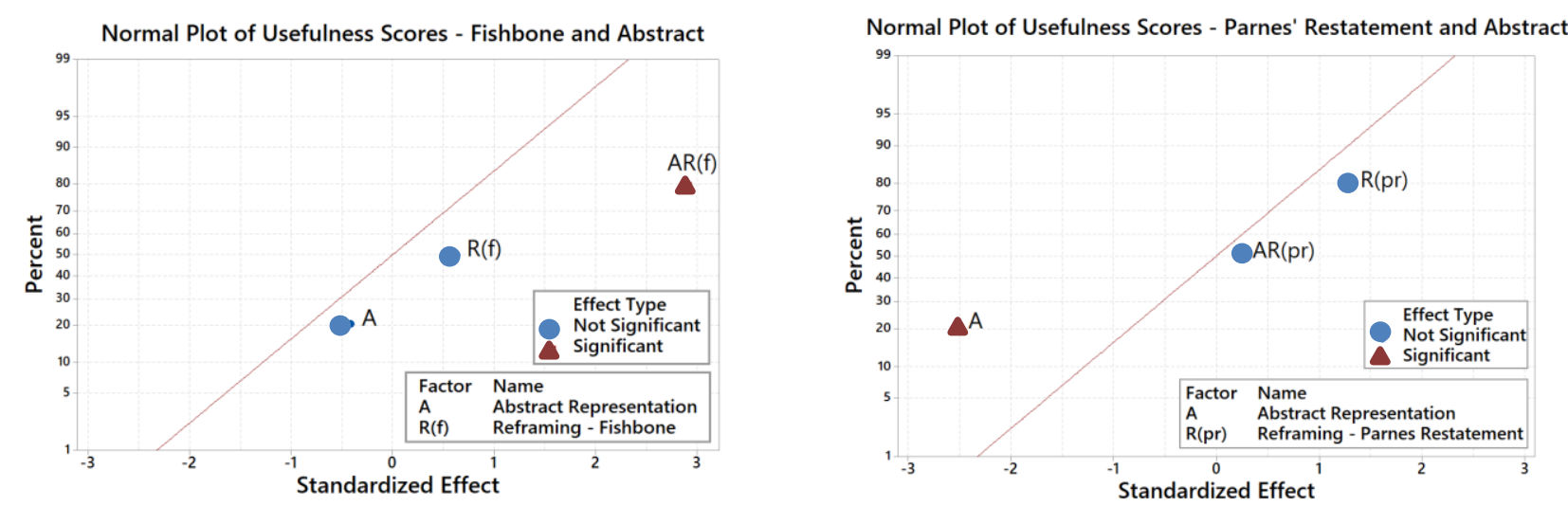

Figure 5. Normal plot of usefulness scores for factors Abstract Representation with Fishbone (left) and Abstract Representation with Parnes' Restatement (right).

\section{DIscussion}

Fishbone diagram encourages a more logical, category-based, cause-and-effect questioning approach at converging on the problem's root while Parnes' restatement/SCAMPER encourages radical divergence in framing problems before ideation. Comparing the two Reframing methods on novelty and usefulness with each other and to the control brief (RQ1) using a two-independent sample t-test showed no difference in both Reframing methods regardless whether the abstraction was present or absent. From the full factorial design of experiments (RQ2), the main effect of Abstract Representation did not have any significant effects on novelty and usefulness. This contrasts with previous studies which showed improved novelty of solutions with abstract stimuli (Heckler, 2010, Zahner et al., 2010). We posit that this is because groups which received the abstract brief alone may be passive recipients of examples of abstraction and were not instructed to re-represent or challenge the requirements, i.e. during C-Sketch ideation, these groups worked on the given brief instead of a reframed statement, unlike the Reframing groups who were formally guided through a process of re-representing the problem and worked on their own reframed statement during Csketch ideation. While the main effect of both Fishbone diagram and Parnes' restatement method did not result in higher usefulness scores, there is positive two-way interaction effects of these reframing methods with Abstract Representation on both novelty and usefulness scores (aside from Parnes' and Abstract having no interaction effect on usefulness). This may suggest that it is neither the Abstract Representations themselves that help, nor the problem framing with either logical questioning or divergence using substitution approaches initially but rather the active teleological re-representation of the problem for themselves assisted with abstract functional stimuli that helps designers think of more novel solutions. We suggest that challenges to transfer (Helms and Goel, 2014) from abstract function stimuli to problem opportunity aid in the reframing of the design problem and improve the novelty of sketches. Using the FBS (Function, Behaviour, Structure) model (Gero and Mc Neill, 1998), we postulate that Abstract Representations (using troponyms (action verbs) and hypernyms (generic action terms)) given as stimuli 
help train abstraction in thinking and encourage modifications/transformations in "action" and function (rather than behaviour and structure) in the reframed opportunity statement (Sarkar and Chakrabarti, 2011). Finally, we suggest that reflection, shifting perspectives and re-representing problem opportunities at higher levels of abstraction helps designers to cross domains, reduce fixation and thereby improve novelty scores of the concept sketches.

\section{CONCLUSION AND FUTURE WORK}

The research aimed to determine the effect of the Reframing methods with abstract stimuli on the novelty and usefulness of ideas. The key finding is that the presence of both abstract stimuli and either one of the Reframing methods yielded significantly higher novelty scores with medium to large effect sizes and fishbone with abstract stimuli yielded significantly higher usefulness scores; this discovery was made possible because of the use of a full factorial DOE. Interestingly, comparing both Reframing methods, creativity scores did not differ significantly. Future studies can be improved by increasing the sample size and involving participants from the design profession to support the findings of this paper outside the academic setting. Future studies will also look deeper into why and how Reframing and abstract stimuli interact to influence creativity scores by analysing the participant input from the Reframing exercises. Using problem maps (Dinar et al., 2011) with semantic analysis of relationships between the "How Might We" frames to solutions, we could go deeper into understanding cognitive mechanisms and uncover more in-depth insights into influences on solution creativity. We believe these findings are helpful for educators and design practitioners seeking ways to foster creativity and innovation in design.

\section{ACKNOWLEDGEMENTS}

This research project is supported by the Singapore University of Technology and Design (SUTD) and the SUTD-MIT International Design Centre (IDC, idc.sutd.edu.sg). The authors thank Christine Yogiaman and the 2018 batch of first-year SUTD student-designers for their ideation efforts and collaboration.

\section{REFERENCES}

Amabile, T.M. (1996), Creativity in Context, Westview Press, Boulder, CO.

Antony, J. (2014), Design of Experiments for Engineers and Scientists, Elsevier Ltd, London.

Bourgeois-Bougrine, S., Buisine, S., Vandendriessche, C., Glaveanu, V. and Lubart, T. (2017), "Engineering students' use of creativity and development tools in conceptual product design: What, when and how?" Thinking Skills and Creativity, Vol. 24, pp. 104-117.

Camburn, B.A., Auernhammer, J.M., Sng, K.H.E., Mignone, P.J., Arlitt, R.M., Perez, K.B., Huang, Z., Basnet, S., Blessing, L.T. and Wood, K.L. (2017), Design Innovation: A Study of Integrated Practice. No. 58219, p. V007T006A031.

Carlgren, L., Rauth, I. and Elmquist, M. (2016), "Framing Design Thinking: The Concept in Idea and Enactment", Creativity and Innovation Management, Vol. 25 No. 1, pp. 38-57.

Dean, D.L.H., Jillian M.; Rodgers, T.L.; and Santanen, E.L. (2006), "Bucknell University. Identifying Quality, Novel, and Creative Ideas: Constructs and Scales for Idea Evaluation", Journal of the Association for Information Systems, Vol. 7 No. 10, pp. 646-699.

Dinar, M., Shah, J., Hunt, G., Campana, E. and Langley, P. (2011), Towards a Formal Representation Model of Problem Formulation in Design. No. 54860, pp. 263-272.

Finke, R.A. (1990), Creative imagery: Discoveries and inventions in visualization, Lawrence Erlbaum Associates, Inc, Hillsdale, NJ, US.

Fogler, H.S. and LeBlanc, S.E. (2008), Strategies for creative problem solving, Pearson Education, Upper Saddle River, NJ. Gero, J.S. and Mc Neill, T. (1998), "An approach to the analysis of design protocols", Design Studies, Vol. 19 No. 1, pp. 21-61.

Getzels, J.W. (1975), "Problem-Finding and the Inventiveness of Solutions", The Journal of Creative Behavior, Vol. 9 No. 1, pp. 12-18.

Getzels, J.W. and Csikszentmihalyi, M. (1977), "The Creative Vision: A Longitudinal Study of Problem Finding in Art", Journal of Aesthetics and Art Criticism, Vol. 36 No. 1.

Goldstone, R.L. and Sakamoto, Y. (2003), "The transfer of abstract principles governing complex adaptive systems", Cogn Psychol, Vol. 46 No. 4, pp. 414-466.

Heckler, A.F. (2010), Concrete vs. abstract problem formats: a disadvantage of prior knowledge. p. 365-371.

Helms, M. and Goel, A.K. (2014), The Four-Box Method of Problem Specification and Analogy Evaluation in Biologically Inspired Design. No. 46407, p. V007T007A005. 
Hicks, M.J. (2004), Problem Solving and Decision Making: Hard, Soft and Creative Approaches, 2nd Edition, Thomson Learning, London.

Higgins, J.S., Maitland, G.C., Perkins, J.D., Richardson, S.M. and Piper, D.W. (1989), "Identifying and solving problems in engineering design", Studies in Higher Education, Vol. 14 No. 2, pp. 169-181.

Holyoak, K.J. and Thagard, P. (1989), “Analogical Mapping by Constraint Satisfaction”, Cognitive Science, Vol. 13 No. 3, pp. 295-355.

Ishikawa, K. (1982), Guide to quality control,

Josephson, J.R.J. and Susan G. (1994), Abductive inference : computation, philosophy, technology, Cambridge University Press, Cambridge, New York.

Kaufman, J.C., Baer, J., Cole, J.C. and Sexton*, J.D. (2008), “A Comparison of Expert and Nonexpert Raters Using the Consensual Assessment Technique”, Creativity Research Journal, Vol. 20 No. 2, pp. 171-178.

Kepner, C.H. and Tregoe, B.B. (2013), The new rational manager : an updated edition for a new world,

Kohfeldt, D. and Day Langhout, R. (2012), The Five Whys Method: A Tool for Developing Problem Definitions in Collaboration with Children. http://doi.org/10.1002/casp.1114.

Kruger, C. and Cross, N. (2006), "Solution driven versus problem driven design: strategies and outcomes", Design Studies, Vol. 27 No. 5, pp. 527-548.

Linsey, J.S. (2007), Design-by-analogy and representation in innovative engineering concept generation, in Mechanical Engineering. University of Texas at Austin. p. 393.

Linsey, J.S., Markman, A.B. and Wood, K.L. (2012), "Design by Analogy: A Study of the WordTree Method for Problem Re-Representation”, Journal of Mechanical Design, Vol. 134 No. 4, 041009-041009-041012.

Linsey, J.S., Tseng, I., Fu, K., Cagan, J., Wood, K.L. and Schunn, C. (2010), “A Study of Design Fixation, Its Mitigation and Perception in Engineering Design Faculty”, Journal of Mechanical Design, Vol. 132 No. 4, pp. $1-12$.

Linsey, J.S., Wood, K.L. and Markman, A.B. (2008), "Modality and representation in analogy", Artificial Intelligence for Engineering Design, Analysis and Manufacturing, Vol. 22 No. 2, pp. 85-100.

Marschark, M. and Paivio, A. (1977), "Integrative processing of concrete and abstract sentences", Journal of Verbal Learning and Verbal Behavior, Vol. 16 No. 2, pp. 217-231.

Moreno, D.P., Blessing, L.T., Yang, M.C., Hernández, A.A. and Wood, K.L. (2016), “Overcoming design fixation: Design by analogy studies and nonintuitive findings", Artificial Intelligence for Engineering Design, Analysis and Manufacturing, Vol. 30 No. 2, pp. 185-199.

Parnes, S.J. (1967), Creative Behavior Guidebook, Charles Scribner's Sons, New York.

Sarkar, P. and Chakrabarti, A. (2011), "Assessing design creativity”, Design Studies, Vol. 32 No. 4, pp. 348-383.

Schön, D.A. (1988), "Designing: Rules, types and worlds”, Design Studies, Vol. 9 No. 3, pp. 181-190.

Schön, D.A. (1983), The reflective practitioner : how professionals think in action, Basic Books, New York.

Serrat, O. (2017), The SCAMPER Technique. http://doi.org/10.1007/978-981-10-0983-9_33.

Shah, J.J., Smith, S.M. and Vargas-Hernandez, N. (2003), "Metrics for measuring ideation effectiveness", Design Studies, Vol. 24 No. 2, pp. 111-134.

Shah, J.J., Vargas-Hernandez, N.O.E., Summers, J.D. and Kulkarni, S. (2011), "Collaborative Sketching (CSketch) - An Idea Generation Technique for Engineering Design”, The Journal of Creative Behavior, Vol. 35 No. 3, pp. 168-198.

Storme, M., Myszkowski, N., Çelik, P. and Lubart, T. (2014), "Learning to judge creativity: The underlying mechanisms in creativity training for non-expert judges", Learning and Individual Differences, Vol. 32, pp. 19-25.

Studer, J., McKilligan, S., R Daly, S. and Seifert, C. (2016), Cognitive Heuristics in Defining Engineering Design Problems. http://doi.org/10.1115/DETC2016-59942.

Tversky, B. and Suwa, M. (2009), Thinking with Sketches.

Valkenburg, A.C. (2000), The reflective practice in product design teams, in None (EN).

Watson, P. Rules of thumb on magnitudes of effect sizes. Available: http://imaging.mrccbu.cam.ac.uk/statswiki/FAQ/effectSize [Accessed].

Zahner, D., Nickerson, J.V., Tversky, B., Corter, J.E. and Ma, J. (2010), “A fix for fixation? Rerepresenting and abstracting as creative processes in the design of information systems", Artificial Intelligence for Engineering Design, Analysis and Manufacturing, Vol. 24 No. 2, pp. 231-244. 